\title{
A promoter variant in ZNF804A decreasing its expression increases the risk of autism spectrum disorder in the Han Chinese population
}

\author{
Linna Zhang ${ }^{1}$, Yue Qin ${ }^{2,3}$, Xiaohong Gong ${ }^{2,3}$, Rui Peng ${ }^{2,3}$, Chunquan Cai ${ }^{4}$, Yufang Zheng $\mathbb{1}^{2}$, Yasong Du ${ }^{1}$ and \\ Hongyan Wang $2,5,6$
}

\begin{abstract}
Synaptic pathology may be one of the cellular substrates underlying autism spectrum disorder (ASD). ZNF804A is a transcription factor that can affect or regulate the expression of many candidate genes involved in ASD. It also localizes at synapses and regulates neuronal and synaptic morphology. So far, few reports have addressed possible associations between ZNF804A polymorphisms and ASD. This study aimed to investigate whether ZNF804A genetic variants contribute to ASD susceptibility and its possible pathological role in the disorder. We analyzed the relationship of two polymorphisms (rs10497655 and rs34714481) in ZNF804A promoter region with ASD in 854 cases versus 926 controls. The functional analyses of rs 10497655 were then performed using real-time quantitative polymerase chain reaction, electrophoretic mobility shift assays, chromatin immunoprecipitation and dual-luciferase assays. The variant rs 10497655 was significantly associated with ASD $(P=0.007851)$, which had a significant effect on ZNF804A expression, with the T risk allele homozygotes related with reduced ZNF804A expression in human fetal brains. HSF2 acted as a suppressor by down-regulating ZNF804A expression and had a stronger binding affinity for the T allele of rs 10497655 than for the $C$ allele. This was the first experiment to elucidate the process in which a disease-associated SNP affects the level of ZNF804A expression by binding with the upstream regulation factor HSF2. This result indicates that the rs10497655 allelic expression difference of ZNF804A during the critical period of brain development may have an effect on postnatal phenotypes of ASD. It reveals new roles of ZNF804A polymorphisms in the pathogenesis of psychiatric disorders.
\end{abstract}

\section{Introduction}

Autism spectrum disorder (ASD) is characterized by early-onset deficiencies in social interactions as well as restricted and repetitive behaviors, interests or activities ${ }^{1}$.

\footnotetext{
Correspondence: Yasong Du (yasongdu@163.com) or Hongyan Wang (wanghylab@fudan.edu.cn)

'Department of Child \& Adolescent Psychiatry, Shanghai Mental Health Center, Shanghai Jiao Tong University School of Medicine, Shanghai 200030, China

${ }^{2}$ Obstetrics and Gynecology Hospital, State Key Laboratory of Genetic Engineering at School of Life Sciences, Institute of Reproduction and

Development, Fudan University, Shanghai 200011, China

Full list of author information is available at the end of the article.

These authors contributed equally: Linna Zhang, Yue Qin, Xiaohong Gong
}

According to a report from the United States in 2012, the prevalence of this pervasive developmental disorder has increased to 1 in 68 children $^{2}$. The Centers for Disease Control and Prevention (CDC of the USA) reported that ASD occurs in all racial, ethnic, and socioeconomic groups but is approximately 4.5 times more common among boys than among girls. ASD is a neurodevelopmental disorder, with an estimated heritability of $0.7-0.9$ based on large-scale studies ${ }^{3,4}$. The genetic etiology of ASD has been proven to be complex and heterogeneous, with over 800 genes implicated in this disease (https:// www.sfari.org/resource/sfari-gene/), as shown by various 
studies including genome-wide association studies $(\text { GWAS })^{5,6}$, whole exome sequencing (WES) $)^{7,8}$, association studies or functional analyses of candidate genes for single nucleotide polymorphisms $(\mathrm{SNP})^{9-11}$ or rare mutations ${ }^{12,13}$ and the detection of copy number variations $(\mathrm{CNV})^{14,15}$.

Increasing evidence shows that synaptic pathology may be one of the cellular substrates underlying $\mathrm{ASD}^{16}$. Recently, it has been found that ZNF804A/Zfp804A localizes at synapses and regulates neuronal and synaptic morphology ${ }^{17}$. ZNF804A is a gene that encodes a transcription factor which contains zinc finger and nucleic acid binding domains. It can affect or regulate the expression of many candidate genes involved in ASD, such as $P D E 4 B$ and $D R D 2^{18-21}$. Moreover, the expression of ZNF804A in the brain has been revealed to be significantly decreased in individuals with ASD than con$\operatorname{trols}^{22}$. ZNF804A was also found to be a risk gene for ASD by $\mathrm{CNV}$ analyses ${ }^{23-25}$. Therefore, the identification and evaluation of correlation between ZNF804A and ASD is of great value. So far, few reports have addressed possible associations between ZNF804A polymorphisms and ASD, except that Anitha and colleagues reported the intronic SNP rs7603001 of ZNF804A was related to verbal skills in individuals with $\mathrm{ASD}^{22}$. It is necessary to investigate more potential ASD-associated variations in the ZNF804A gene.

In order to determine whether ZNF804A genetic variants in the promoter contribute to ASD susceptibility and its possible pathological role in the disorder, two common polymorphisms with minor allele frequency (MAF) $>10 \%$ in the promoter region were tested for association with ASD in 854 Chinese ASD cases and 926 controls. Our results demonstrated that rs 10497655 was associated with ASD susceptibility and the $T$ allele significantly increases the risk of ASD. Furthermore, functional assays were performed to explore its role of rs10497655 in the pathogenesis of this disease.

\section{Materials and Methods}

\section{Ethics statement}

Written informed consent was obtained from the controls and the guardians or the parents of the children with ASD. This study was approved by the Ethics Committee of the Shanghai Mental Health Center, Shanghai Jiao Tong University School of Medicine and the Ethics Committee of the School of Life Sciences, Fudan University. Ethical approval for the collection and the distribution of the brain samples for research was obtained from Tianjin Children's Hospital.

\section{Study subjects}

Samples of 854 patients with ASD (751 males and 103 females) and 926 controls (817 males and 109 females) were recruited separately from Department of Child and Adolescent Psychiatry, Shanghai Mental Health Center, Shanghai Jiao Tong University School of Medicine (Shanghai, China) and Fudan University (Shanghai, China) between February 2010 and January 2014. All of the subjects collected were ethnic Han Chinese. All of the controls recruited from Fudan University were matched to the patients according to geography and gender. The average ages (years) of the ASD patients and the controls were $5.91 \pm 0.15$ and $18.58 \pm 0.01$ (mean $\pm \mathrm{SE}$ ), respectively. Of the patients, 670 individuals recruited from 2010 to 2013 were diagnosed with ASD according to the criteria of Diagnostic and Statistical Manual of Mental Disorders, fourth Edition (DSM-4) of the American Psychiatric Association (APA) and 184 individuals recruited from 2013 to 2014 were diagnosed with ASD according to DSM- $5^{1}$. Based on the DSM- 4 or DSM- 5 scores on overall levels of social ability (scores of $0-4$ for DSM-4 and scores of 0-3 for DSM-5), repetitive behavior (scores of 0-4 for DSM-4 and DSM-5) or language (scores of 0-4 for DSM-4 only), individuals with ASD were divided into mild deficiency (scores of 0,1 , or 2) subgroups and severe deficiency (scores of 3 or 4 ) subgroups.

\section{Genotyping of two common variants in the ZNF804A gene}

Between 1 and $3 \mathrm{~mL}$ of venous blood was collected from each individual in this study. Genomic DNA was isolated using the Mammalian Genomic DNA Extraction Kit (Lifefeng, Shanghai, China). Two promoter SNPs with MAF $>10 \%$ (rs10497655 and rs34714481) were genotyped by sequencing in 854 ASD cases and 926 controls. All primer pairs for sequencing were designed using the online software PRIMER 3 (http://bioinfo.ut.ee/ primer3-0.4.0/) and are shown in Table S1. For the sequencing reaction, PCR products were pre-treated with a mixture of 10 units of Exo I and 1 unit of FastAP (Thermo Scientific, MA, USA). BigDye Terminator v3.1 was used for the direct sequencing reaction according to the manufacturer's instructions (Applied Biosystems, CA, USA). HiDi formamide (Applied Biosystems, CA, USA) was used in the subsequent denaturation, and finally, the samples were submitted for sequencing using an $\mathrm{ABI}$ 3730xl sequencer (Applied Biosystems, CA, USA). The results were analyzed with Genalys software (version 2.8.3).

\section{Real-time quantitative polymerase chain reaction (RT- qPCR) in brain samples}

Eighteen human fetal brain tissues from terminations of pregnancy were provided by Tianjin Children's Hospital. All were second-trimester fetal brain samples that had no obvious defects. Genomic DNA and total RNA were extracted simultaneously from all samples using the 
TRNzol reagent (TIANGEN BIOTECH, Beijing, China). Each DNA sample was genotyped for the SNP rs10497655. The primer pairs are listed in Table S1.

RNA samples were treated with gDNase for $3 \mathrm{~min}$ to remove genomic contamination and then first-stranded cDNA was synthesized using the FastQuant RT kit (with gDNase) (TIANGEN BIOTECH, Beijing, China). ZNF804A mRNA levels were measured by quantitative real-time PCR using the StepOne system (Applied Biosystems, CA, USA). The standard reaction mixture contained $10 \mu \mathrm{M}$ of each primer, the SuperReal PreMix Plus (SYBR Green) kit (TIANGEN BIOTECH, Beijing, China) and $2 \mu \mathrm{l}$ of cDNA (1:10 dilution). $h G A P D H$ was used as an internal reference gene. Relative ZNF804A mRNA levels were measured independently with two pairs of primers targeting different regions. All of the samples were finished for RT-qPCR with oligonucleotides hZNF804A-F1/ $R 1$. Each reaction was performed in triplicate at least four times. The primers are listed in Table S1.

\section{Electrophoretic mobility shift assays (EMSA)}

Nuclear proteins were extracted from HEK-293T cells using NE-PER nuclear and cytoplasmic extraction reagents (Pierce, IL, USA). Duplex oligonucleotide probes representing the $-1052 \mathrm{C}$ or $\mathrm{T}$ alleles of rs10497655 (sequences listed in Table S1) were labeled with biotin. The assays were performed using a LightShift Chemiluminescent EMSA kit (Thermo Scientific, MA, USA) according to the manufacturer's protocols. Briefly, 50 fmols of biotin-labeled duplex oligonucleotides bearing either the $-1052 \mathrm{C}$ or the $\mathrm{T}$ allele of rs 10497655 were incubated with $5 \mu \mathrm{g}$ of nuclear extracts for $20 \mathrm{~min}$ in $10 \times$ binding buffer supplemented with $1 \mu \mathrm{g} / \mu \mathrm{l}$ poly (dI.dC), $50 \%$ glycerol and $1 \%$ NP- 40 . Unlabeled probes at 5 - or $50-$ fold molar excesses, as indicated, were added to the reaction for competition. The reaction mixture was then electrophoresed on a native $6 \%$ polyacrylamide gel and transferred to a positive nylon membrane. The detection of biotin-labeled DNA was performed using stabilized streptavidin-horseradish peroxidase conjugate nd the membrane was exposed to X-ray film. Each experiment was repeated three times.

\section{Chromatin immunoprecipitation (ChIP) assays}

Human neuroblastoma IMR-32 cells were grown in DMEM supplemented with 10\% FBS (Gibco, CA, USA) and 1\%oo Plasmocin (InvivoGen, CA, USA). ChIP assays were performed using nuclear extracts obtained from IMR-32 cells heterozygous for $\mathrm{C}>\mathrm{T}$ at rs10497655 in the ZNF804A promoter. For each reaction, approximately $2 \times$ $10^{6}$ IMR-32 cells were cross-linked with $1 \%$ formaldehyde at room temperature for $10 \mathrm{~min}$, followed by termination with glycine added to the sample for another $5 \mathrm{~min}$. The chromatin was then sonicated into fragments with a length of 200 to $750 \mathrm{bp}$ using Bioruptor (Diogenode, Liege, Belgium). For immunoprecipitation, the sheared chromatin was incubated with anti-HSF2 (sc-13056x) antibody (Santa Cruz Biotechnology, CA, USA) or nonspecific rabbit IgG (Invitrogen, CA, USA) for $4 \mathrm{~h}$ at $4{ }^{\circ} \mathrm{C}$. Finally, the purified DNA fragments were identified by PCR (primers see Table S1) that caught a 254-bp-specific product that covered the variant $\mathrm{C}>\mathrm{T}$. To further investigate the difference in the binding affinity between the $C$ and $T$ alleles of the SNP rs10497655, the ratio of the two alleles was quantified using SNaPshot from the ChIP input and the purified DNA precipitated with anti-HSF2. The SNaPshot products were detected on an ABI 3730xl automated sequencer and analyzed with Peak scanner software (version 1.0). All values were normalized to the input levels. Three independent assays were performed.

\section{Plasmid construction}

To construct the ZNF804A promoter luciferase reporter plasmid, the 1148-bp fragment from -1715 to -568 of human ZNF804A was amplified from genomic DNA using PCR, which contains the $\mathrm{T}$ allele of the $-1052 \mathrm{C}>\mathrm{T}$ SNP (rs10497655). The PCR products were subcloned into the $M l u \mathrm{I}$ and $\mathrm{XhoI}$ restriction sites of the pGL3-Basic vector (Promega, WI, USA), in which the firefly luciferase gene was used as a reporter. The corresponding plasmid containing the $\mathrm{C}$ allele was generated by site-directed mutagenesis using the MutanBEST kit (Takara, Dalian, China). Human HSF2 cDNA was donated by Professor Han (Xiamen University, Xiamen, China). The HSF2 CDS fragments were amplified with KOD PLUS (Toyobo, Shanghai, China) and cloned into the EcoRV and KpnI restriction sites of the pcDNA3.1(-) vector (Invitrogen, CA, USA).We verified all of the plasmids by bidirectional sequencing. The primers used are shown in Table S1.

\section{Cell culture, transfection and luciferase reporter assays}

Human embryonic kidney HEK-293T cells and human neuroblastoma SK-N-AS cells were grown in DMEM supplemented with 10\% FBS (Corning, NY, USA) and $1 \%$ oo Plasmocin (InvivoGen, CA, USA). For the ZNF804A promoter activity study, 80\% confluent HEK-293T and SK-N-AS cells in the 24-well plate were co-transfected with $500 \mathrm{ng}$ of ZNF804A promoter reporter plasmid carrying the $\mathrm{C}$ or the $\mathrm{T}$ allele of rs10497655 and $10 \mathrm{ng}$ of the pRL-TK plasmid (Promega, WI, USA), in which the Renilla luciferase gene was used as an internal control. Furthermore, the cells were additionally co-transfected with $200 \mathrm{ng}$ of the pcDNA3.1(-)-HSF2 expression plasmid or equivalent amounts of the empty pcDNA3.1(-) vector. Lipofectamine 2000 or 3000 (Invitrogen, CA, USA) transfection reagent was used for the transfections, according to the manufacturer's instructions. After $24 \mathrm{~h}$ of culture, the transfected cells were lysed and $20 \mu$ of the 
Table 1 Allele frequencies of two SNPs in the ASD patients and controls

\begin{tabular}{|c|c|c|c|c|c|c|c|c|c|}
\hline \multirow[t]{2}{*}{ SNP ID } & \multirow[t]{2}{*}{ Allele } & \multirow[t]{2}{*}{ Control, $n \%$} & \multirow[t]{2}{*}{ Case, $n \%$} & \multirow[t]{2}{*}{$P$ value $^{a}$} & \multirow[t]{2}{*}{ OR $(95 \% \mathrm{CI})^{b}$} & \multicolumn{4}{|l|}{$\mathrm{MAF}^{\mathrm{c}}$} \\
\hline & & & & & & Control & Case & $\mathrm{CHB}^{\mathrm{d}}$ & $\mathrm{CHS}^{\mathrm{C}}$ \\
\hline \multirow[t]{2}{*}{ rs10497655 } & $C$ & $963(52.0 \%)$ & $811(47.5 \%)$ & 0.007851 & $1.20(1.05-1.37)$ & 0.48 & 0.53 & 0.495 & 0.476 \\
\hline & $\mathrm{T}$ & 889(48.0\%) & $897(52.5 \%)$ & & & & & & \\
\hline \multirow[t]{2}{*}{ rs34714481 } & A & 1234(66.6\%) & 1105(64.7\%) & 0.2381 & $1.08(0.95-1.24)$ & 0.33 & 0.35 & 0.286 & 0.319 \\
\hline & G & 618(33.4\%) & $603(35.3 \%)$ & & & & & & \\
\hline
\end{tabular}

$O R$ odds ratio, $\mathrm{Cl}$ confidence interval

aDifferences between cases and controls under allele frequencies were compared using the chi-square test with $1 \mathrm{degree}$ of freedom (df)

${ }^{b}$ ORs and $95 \%$ Cls were calculated by the logistic regression analysis

${ }^{c} \mathrm{MAF}$ indicates minor allele frequency

${ }^{d}$ They represent allelic frequencies in the Chinese Han populations based on data from the 1000 Genomes Project

supernatant was assayed for luciferase activity using the Dual-Luciferase Reporter Assay System (Promega, WI, USA). The relative reporter activity was obtained by normalization of the firefly activity to Renilla activity. Each assay was performed in triplicate, and each experiment was performed at least three times.

\section{Bioinformatics analysis}

Potential transcription factors (TFs) that bind the sites around the specific target SNP rs10497655 were computationally predicted using the online software TFSEARCH (http://www.cbrc.jp/research/db/TFSEARCH.html).

\section{Statistical analysis}

The statistical analysis was conducted with $\mathrm{R}$ software (Version 3.1.3) and GraphPad Prism software (Version 5.04, GraphPad Software Inc., USA), which was also used for graphics. Differences in allelic or genotypic frequencies between the ASD cases and the controls were compared by the $\chi^{2}$ test, which was also used to assess the Hardy-Weinberg equilibrium (HWE) for the controls. To evaluate the associations between genotypes and ASD risk, odds ratios (ORs) and 95\% confidence intervals (CIs) were calculated using a logistic regression analysis. The online software SNPStats (http://bioinfo.iconcologia.net/ snpstats/start.htm) was used to analyze the association between different haplotypes and ASD risk. In RT-qPCR, fold changes were calculated using the $2^{-\Delta \Delta C t}$ method and presented as normalized fold expression. The Mann-Whitney $U$ test was used to evaluate the differences in ZNF804A relative expression levels between the two defined groups. Student's $t$-test was used to compare the values of SNaPshot assays. A one-way ANOVA test was applied for luciferase assays and co-transfection. A repeated-measures ANOVA test was also used in the assays co-transfected with the HSF2 plasmid or the pcDNA3.1(-) empty vector. In graphics, the quantitative variables are given as the mean $\pm \mathrm{SE}$. All statistical tests were two-tailed, with a significance level of 0.05 .

\section{Results \\ The ZNF804A promoter variant rs10497655 C > T significantly increases the risk of ASD}

We searched for common genetic variants with MAF > $10 \%$ and identified two SNPs (rs10497655 and rs34714481) within the promoter region of ZNF804A in the case-control cohort. We analyzed the relationship of rs10497655 or rs34714481 with the risk of ASD in 854 cases versus 926 controls. The genotype distribution of two SNPs were in Hardy-Weinberg equilibrium in controls. It was showed that strong association with ASD was observed for the SNP rs10497655 ( $P=0.007851$, Table 1$)$. The minor $\mathrm{T}$ allele was significantly related to a higher risk for $\operatorname{ASD}(\mathrm{OR}=1.20,95 \% \mathrm{CI}=1.05-1.37)$. On the other hand, no association was detected between SNP rs34714481 and ASD $(P=0.2381)$. The genotype frequencies of rs10497655 showed significant differences between ASD cases and controls in codominant, recessive and overdominant models $\left(P=2.12 \times 10^{-5}, P=2.23 \times\right.$ $10^{-5}, P=1.35 \times 10^{-4}$, respectively, Table 2). Compared with the CC and CT genotypes, the TT genotype of rs10497655 was significantly more common in the ASD cases than in the controls $(\mathrm{OR}=1.58,95 \% \mathrm{CI}=$ 1.28-1.95). Individuals with ASD were divided into mild deficiency and severe deficiency categories based on social ability, language and repetitive behaviors, separately. A stronger association of the SNP rs10497855 was observed in ASD individuals with severe deficiency on language $(P$ $=0.0006$, OR $=1.37,95 \% \mathrm{CI}=1.14-1.63$, Table 3 ), whereas no association was observed in other categories considering multiple test corrections (Table 3). Compared with the CC-CT genotypes, the TT genotype was significantly more common in ASD individuals with severe deficiency on language than in the controls $(P=1.00 \times$ $\left.10^{-4}, \quad \mathrm{OR}=1.90, \quad 95 \% \quad \mathrm{CI}=1.45-2.50\right)$. No gender 
differences were observed for allele frequencies of rs10497655 between controls and cases when males or females were considered separately (data was not shown).

\section{The rs10497655 C > T variation decreases the} transcriptional activity of ZNF804A in vivo

Since rs10497655 was located in the promoter region of the ZNF804A gene, different alleles may influence the transcription level of ZNF804A mRNA. To test this possibility, 18 post-mortem fetal brain samples from secondtrimester abortions were tested for in vivo mRNA expression levels, with 8 CC genotypes, 6 CT genotypes and 4 TT genotypes. The promoter activity of the TT group was significantly lower than the $\mathrm{CC}+\mathrm{CT}$ genotype group, which displayed approximately forty percent of the latter (Fig. 1). The relative ZNF804A mRNA levels of the brain samples were measured independently with two pairs of primers using the RT-qPCR method, and the two sets of data showed a similar trend (see Figure S1).
Combined with the genetic data of rs10497655 in ASD, this result indicates that the effect of the risk $\mathrm{T}$ allele on ASD is mediated by reducing the expression of ZNF804A. This may be induced by the interaction of the rs10497655 $\mathrm{C}>\mathrm{T}$ variation with certain inherent factors inside cells that greatly influence the transcription process of ZNF804A.

\section{The variant rs 10497655 alters the binding capacity with nuclear proteins in the promoter region}

To verify the binding capacity of transcription factors to the $\mathrm{C}$ or $\mathrm{T}$ alleles of rs10497655, EMSA was carried out with nuclear extract prepared from HEK-293T cells. The EMSA analysis showed that the 26-bp core sequence containing the $\mathrm{T}$ allele of rs10497655 had a higher binding affinity than the $\mathrm{C}$ allele (Fig. 2a, lanes 2 and 9). The specificity of the proteins bound to the fragment covering rs10497655 was proven by competition tests in which 5-/ 50 -fold excess of unlabeled corresponding probes were

Table 2 Associations between rs10497655 and ASD in the case-control study

\begin{tabular}{|c|c|c|c|c|c|c|c|}
\hline SNP ID & Genetic model & Pattern & Control, $n \%$ & Case, $n \%$ & $P$ value $^{\mathrm{a}}$ & HWE $P$ val ${ }^{b}$ & OR $(95 \% \mathrm{Cl})^{\mathrm{c}}$ \\
\hline \multirow[t]{9}{*}{ rs10497655 } & \multirow[t]{3}{*}{ Codominant } & $\mathrm{C} / \mathrm{C}$ & $250(27.0 \%)$ & $231(27.0 \%)$ & \multirow[t]{3}{*}{$2.12 \times 10^{-5}$} & \multirow[t]{9}{*}{0.9997} & Reference \\
\hline & & $C / T$ & $463(50.0 \%)$ & $349(40.9 \%)$ & & & $0.82(0.65-1.02)$ \\
\hline & & $\mathrm{T} / \mathrm{T}$ & $213(23.0 \%)$ & 274(32.1\%) & & & $1.39(1.08-1.79)$ \\
\hline & \multirow[t]{2}{*}{ Dominant } & $\mathrm{C} / \mathrm{C}$ & $250(27.0 \%)$ & $231(27.0 \%)$ & \multirow[t]{2}{*}{1} & & Reference \\
\hline & & $\mathrm{C} / \mathrm{T}-\mathrm{TT}$ & 676(73.0\%) & $623(73.0 \%)$ & & & $1.00(0.81-1.23)$ \\
\hline & \multirow[t]{2}{*}{ Recessive } & $\mathrm{C} / \mathrm{C}-\mathrm{C} / \mathrm{T}$ & $713(77.0 \%)$ & $580(67.9 \%)$ & \multirow[t]{2}{*}{$2.23 \times 10^{-5}$} & & Reference \\
\hline & & $\mathrm{T} / \mathrm{T}$ & $213(23.0 \%)$ & 274(32.1\%) & & & $1.58(1.28-1.95)$ \\
\hline & \multirow[t]{2}{*}{ Overdominant } & $\mathrm{C} / \mathrm{C}-\mathrm{T} / \mathrm{T}$ & $463(50.0 \%)$ & 505(59.1\%) & \multirow[t]{2}{*}{$1.35 \times 10^{-4}$} & & Reference \\
\hline & & $C / T$ & $463(50.0 \%)$ & $349(40.9 \%)$ & & & $0.69(0.57-0.83)$ \\
\hline
\end{tabular}

${ }^{a}$ Differences between cases and controls under different genetic models were compared using the chi-square test with 2 degrees of freedom (df) or $1 \mathrm{degree}$ of freedom $(\mathrm{d} f)$

${ }^{\mathrm{b}}$ HWE $P$ val indicates $P$ value for the Hardy-Weinberg equilibrium test in the control subjects

${ }^{\circ} \mathrm{OR}$ indicates the odds ratio; $\mathrm{Cl}$, confidence interval. ORs and $95 \% \mathrm{Cls}$ were calculated by the logistic regression analysis

Table 3 Allele frequencies of rs10497655 in the controls and ASD subgroups

\begin{tabular}{llllllll}
\hline Allele & Control, $\mathbf{n} \%$ & MDS $^{\mathbf{a}}$ & SDS $^{\mathbf{b}}$ & MDR $^{\mathbf{c}}$ & SDR $^{\mathbf{d}}$ & MDL $^{\mathbf{e}}$ & SDL $^{\mathbf{f}}$ \\
\hline $\mathrm{C}$ & $963(52.0 \%)$ & $263(48.2 \%)$ & $488(48.1 \%)$ & $533(48.7 \%)$ & $218(46.8 \%)$ & $286(50.7 \%)$ & $291(44.2 \%)$ \\
$\mathrm{T}$ & $889(48.0 \%)$ & $283(51.8 \%)$ & $526(51.9 \%)$ & $561(51.3 \%)$ & $248(53.2 \%)$ & $278(49.3 \%)$ & $367(55.8 \%)$ \\
P value $^{\#}$ & & 0.1157 & 0.0475 & 0.0856 & 0.0441 & 0.5918 & 0.0006 \\
OR $(95 \% ~ C l) *^{*}$ & & $1.17(0.96-1.41)$ & $1.17(1.00-1.36)$ & $1.14(0.98-1.32)$ & $1.23(1.01-1.51)$ & $1.05(0.87-1.27)$ & $1.37(1.14-1.63)$ \\
\hline
\end{tabular}

$O R$ odds ratio, $\mathrm{Cl}$ confidence interval

\#Differences between cases and controls under allele frequencies were compared using the chi-square test with 1 degree of freedom (df)

*ORs and $95 \% \mathrm{Cls}$ were calculated by the logistic regression analysis

a Patients grouped into mild deficiency on social ability

${ }^{b}$ Patients grouped into severe deficiency on social ability

'Patients grouped into mild deficiency on repetitive behavior

${ }^{d}$ Patients grouped into severe deficiency on repetitive behavior

${ }^{e}$ Patients grouped into mild deficiency on language

fPatients grouped into severe deficiency on language 
added (Fig. 2a, lanes 3-4 and 12-13) and un-competition tests in which an unspecific probe was added (Fig. 2a, lane 7 and 14). A cross-competition experiment further confirmed the observation of a higher binding affinity of the

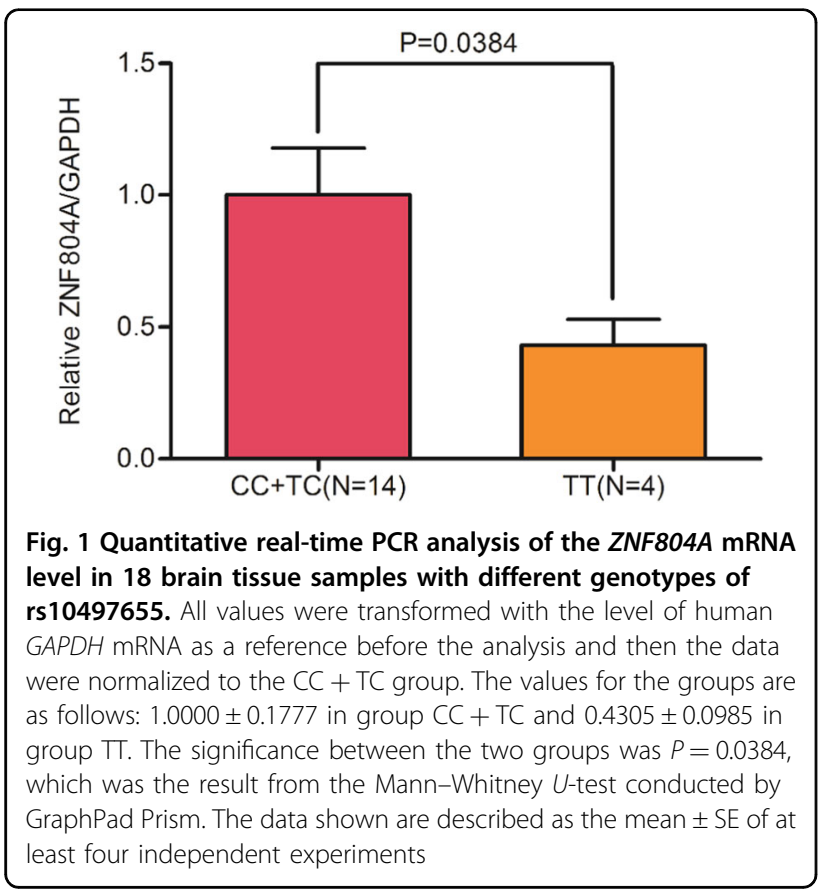

probe containing the $\mathrm{T}$ allele to certain proteins than the $\mathrm{C}$ allele (Fig. 2a, lanes 5-6 and lanes 10-11).

\section{Direct interactions and different Binding Affinities of Transcription factor HSF2 to the C/T alleles of rs 10497655}

The EMSA results revealed that certain TFs bind to the SNP rs10497655 and show different affinities for the T/C alleles. The computational analysis (TFSEARCH) predicted that the alteration from $\mathrm{C}$ to $\mathrm{T}$ at rs10497655 may influence the binding affinity of HSF2 (Figure S2). To prove this prediction, we carried out ChIP assays using IMR-32 cell lines with a heterozygous genotype at rs10497655. The results showed that the region covering the variant rs10497655 was occupied specifically by HSF2 (Fig. 2b). In the quantification by SNaPshot assays, the ratio of $\mathrm{T} / \mathrm{C}$ alleles in the DNA immunoprecipitated by anti-HSF2 (1.4926 \pm 0.1016$)$ was significantly higher than in the input DNA $(P=0.0101)$, indicating that the T allele of rs10497655 had an approximately 1.5-fold higher affinity with HSF2, compared to the $\mathrm{C}$ allele (Fig. 2c).

\section{HSF2 functions as a repressor in the regulation of ZNF804A expression}

Luciferase assays and co-transfection were performed to explore how HSF2 regulates the target promoter sequence. First, we tested the promoter activity of the
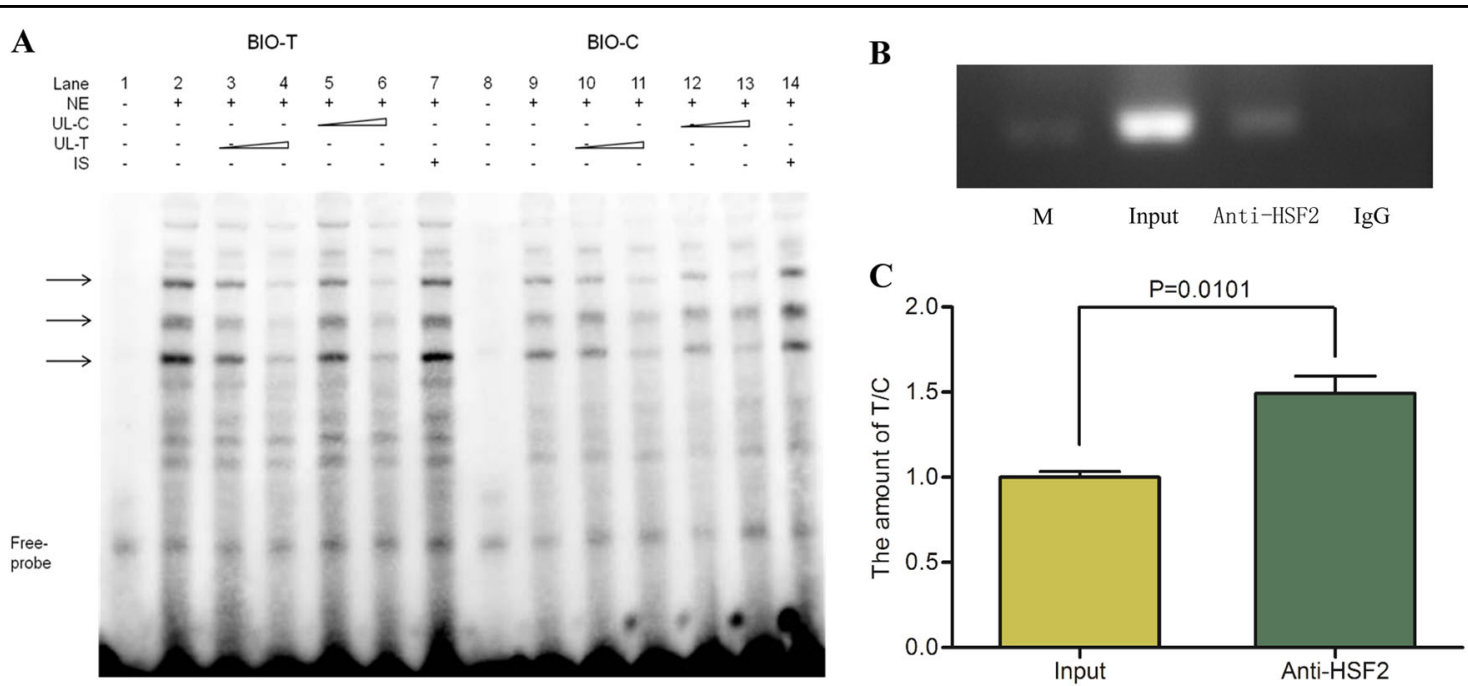

Fig. 2 a The difference in the combining capacity with HEK-293T nuclear proteins in the T and C allele probes shown in EMSA. Lanes 1-7: biotinlabeled probe containing the T allele plus HEK-293T nuclear extract, except for lane 1; lanes 8-14: biotin-labeled probe containing the $\mathrm{C}$ allele and nuclear proteins, except for lane 8; NE represents nuclear proteins isolated from HEK-293T cells; UL-C/T represents unlabeled C/T probes; IS represents the unlabeled probe with an irrelevant sequence. From lane 3 to 4, 5 to 6, 10 to 11, and 12 to 13,5- to 50-fold excesses of the corresponding unlabeled probes were used. $\mathbf{b}$ Chromatin immunoprecipitation (ChIP) assays in IMR-32 cells with the specific antibody to the predicted transcription factor HSF2. The in vivo binding of the HSF2 protein to the rs10497655 position was verified with polymerase chain reaction (PCR). M indicates the 250-bp band of DNA markers; the other three lanes are products from PCR with the ChIP input, DNA precipitated by anti-HSF2 and DNA by IgG as templates. c The amounts of the T/C alleles were quantified by SNaPshot from the ChIP input and products treated anti-HSF2. The ratio of the T allele to the $C$ in the DNA immunoprecipitated $(1.4926 \pm 0.1016)$ was higher than that in the input DNA $(1.0000 \pm 0.0346)$, and the difference reached statistical significance. All values were normalized to the input levels. Significance between two groups was $P=0.0101<0.05$, which was the result from unpaired Student's t-test performed by GraphPad Prism. Data shown are mean \pm SE from three independent assays 

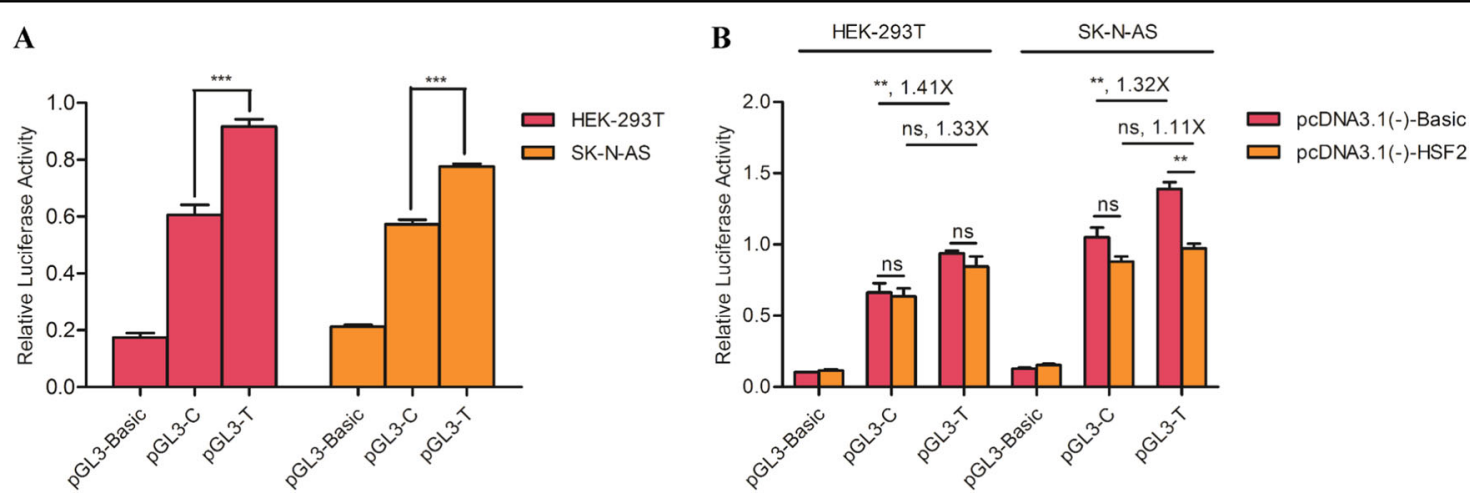

Fig. 3 a Relative luciferase activity in the C or the T allele of the ZNF804A promoter. Luciferase expression was significantly higher in the T allele construct compared with the $C$ allele construct in different cell lines. The values in the HEK-293T cells were as follows: $p G L 3-B a s i c=0.1737 \pm 0.0158$, $p G L 3-C=0.6062 \pm 0.0351, p G L 3-T=0.9174 \pm 0.0250$. The values in the SK-N-AS cells were as follows: $p G L 3-B a s i c=0.2117 \pm 0.0074, p G L 3-C=$ $0.5731 \pm 0.0156, \mathrm{pGL3}-\mathrm{T}=0.7765 \pm 0.0095$. B. HSF2 regulates ZNF804A expression as a repressor by interacting with rs 10497655 loci. a Luciferase construct containing the $\mathrm{C}$ or the T allele was transfected together with pcDNA3.1(-)-Basic (control) or the pcDNA3.1(-)-HSF2 (expression vector) plasmid in different cell lines. In HEK-293T cells, the values of co-transfection with pcDNA3.1(-)-Basic were as follows: pGL3-Basic $=0.1032 \pm 0.0017$, pGL3-C $=0.6633 \pm 0.0649, p G L 3-T=0.9368 \pm 0.0187$, values with pcDNA3.1 $(-)-H S F 2:$ pGL3-Basic $=0.1149 \pm 0.0080, p G L 3-C=0.6359 \pm 0.0553, p G L 3-$ $T=0.8463 \pm 0.0690$. In the SK-N-AS cells, the values of co-transfection with pCDNA3.1 (-)-Basic were as follows: pGL3-Basic $=0.1270 \pm 0.0072, p G L 3-C$ $=1.0515 \pm 0.0673, \mathrm{pGL} 3-\mathrm{T}=1.3898 \pm 0.0482$, values with $\mathrm{pCDNA3} .1(-)-H S F 2: \mathrm{pGL} 3-$ Basic $=0.1529 \pm 0.0087, \mathrm{pGL} 3-\mathrm{C}=0.8784 \pm 0.0378, \mathrm{pGL} 3-\mathrm{T}=$ $0.9729 \pm 0.0337$. Each value is shown as the mean \pm SE. ${ }^{* *}$ Denotes $P<0.001,{ }^{* *}$ Denotes $P<0.01$, ns represents no significance, which were the results from the one-way ANOVA or the repeated measures ANOVA test conducted by GraphPad Prism. The graphs above are representative of three independent experiments

region covering rs10497655 using luciferase assays, in which two cell lines, HEK-293T and SK-N-AS, were transfected with plasmids carrying the $\mathrm{C}$ or $\mathrm{T}$ allele of rs10497655 and an internal control plasmid carrying the Renilla luciferase gene. Both HEK-293T and SK-N-AS cell lines showed that the transcriptional activity of the fragment containing the $\mathrm{T}$ allele of rs10497655 was significantly higher than the $\mathrm{C}$ allele (Fig. 3a). Furthermore, the cells were additionally co-transfected with the pcDNA3.1(-)-HSF2 plasmid or the empty pcDNA3.1(-) vector. When co-transfected with the empty pcDNA3.1(-) vector, the $\mathrm{T}$ allele showed 1.41-fold transcriptional activity compared to the $\mathrm{C}$ allele., Although this divergence decreased, this result was no longer significant when the pcDNA3.1(-)-HSF2 plasmid existed (Fig. 3b). The co-transfection results in human neuroblastoma SKN-AS cells showed that the overexpressed HSF2 decreased the ZNF804A transcription level, and its inhibition effect on the $\mathrm{T}$ allele was stronger than on the $\mathrm{C}$ allele. These data show that HSF2 acts as an inhibitor in ZNF804A transcription. In the RT-qPCR experiment, a lower ZNF804A mRNA level in individuals carrying the TT genotype can partly be explained by the stronger binding affinity and the inhibition effect of HSF2 on the T allele.

\section{Discussion}

Compared with substantial reports of the relationship between $Z N F 804 A$ and schizophrenia $(\mathrm{SCZ})^{26}$, few studies have been reported on ZNF804A and ASD. ZNF804A
CNVs have been found in individuals with $\mathrm{ASD}^{6,22}$, which indicates ZNF804A is involved in ASD. Anitha et al. reported that an intronic SNP rs7603001 was associated with autistic individuals who were verbally deficient. So far, most identified disease-associated SNPs are in introns, causing tremendous difficulties for function studies. To validate and further identify potential functional variations in ZNF804A, we performed an association study in ASD by testing two common SNPs in the promoter region. Our results showed that only one SNP rs10497655 was associated with ASD. What's more, we also found the association of this variant with severe deficiency on language in ASD, consistent with the report of Anitha. The variant rs10497655 has been rarely reported, except in a schizophrenia research study in which no association was found between $\mathrm{rs} 10497655$ and $\mathrm{SCZ}^{27}$. The variant rs10497655 is located 1052 nucleic acids upstream of the transcription start site. A deep exploration was performed to test its potential effect on ZNF804A expression.

ZNF804A expression varies at different brain development times. Hill et al. discovered an allelic expression difference of ZNF804A in second-trimester, but not in first-trimester, human fetal brains, where the schizophrenia risk allele $(\mathrm{T})$ of rs1344706 was associated with reduced ZNF804A expression ${ }^{28}$. The expression of ZNF804A was significantly reduced in specific brain regions in autistic individuals compared with controls ${ }^{22}$. In this study, the transcription levels of ZNF804A were quantified in second-trimester human fetal brains. Genotypes of the positive SNP rs10497655 had a significant 
effect on ZNF804A expression, with the TT homozygotes associated with reduced ZNF804A expression. This result indicates that the allelic expression difference of ZNF804A rs10497655 during the critical period of brain development may have an effect on the postnatal phenotype of ASD.

Although the differences in ZNF804A expression in the ASD cases and the controls and the allelic expression difference of ZNF804A rs1344706 were subsequently reported, the molecular mechanism was not clear. So far, only one report has found that a 5'UTR SNP rs359895 altered the binding affinity of transcription factor $\mathrm{Sp}^{27}$. In our study, EMSA and ChIP assays showed that HSF2 had a stronger binding affinity for the $\mathrm{T}$ allele of rs10497655 than for the $\mathrm{C}$ allele. Luciferase reporter assays showed that HSF2 acted as a suppressor by downregulating $Z N F 804 A$ expression. This is the first report that HSF2 regulates $Z N F 804 A$ expression by binding to a promoter SNP in ZNF804A.

HSF2 belongs to the family of heat shock transcription factors, which mediate cell reactions to stressful conditions. HSF1 is activated by environmental stresses such as heat shock and heavy metals and promotes the synthesis of heat shock proteins, thereby stabilizing intracellular homeostasis $^{29,30}$. HSF2 regulates transcription by forming heterotrimers with HSF1 rather than directly responding to these environmental stresses ${ }^{31}$. The increased level of HSF2 is an activation signal for the following process. HSF2 is involved in corticogenesis and spermatogenesis ${ }^{32}$. Combined with our findings on the effect of HSF2 on ZNF804A expression, we speculate that in the critical stages of brain development, environmental stimuli exert influences on brain development via the HSF2-ZNF804A downstream gene pathway. Umeda-Yano et al., Girgenti et al. and Anitha et al. have confirmed that the upregulation or the knockout of ZNF804A can affect multiple downstream genes, including genes involved in neurodevelopment ${ }^{18,22,33}$. Intriguingly, NLGN4 has been reported to be affected by ZNF804A regulation ${ }^{17}$. The role of NLGN4 in ASD has been widely investigated using genetic, neurodevelopmental and biochemical methods ${ }^{34}$. The temporal and spatial expression characteristics of ZNF804A suggest that it is involved in brain development $^{35}$. The regulation of ZNF804A in a series of genes including NLGN4, may be an important molecular mechanism for ASD. Our research links environmental stimuli and ASD through HSF2-ZNF804A. Obviously, this link is weak, but it provides an exciting new perspective for the understanding of the molecular mechanism of ASD. Experiments are warranted to investigate the alteration of the HSF2 level and its interaction with ZNF804A under various environmental conditions.

Accumulating evidence suggests that common genetic factors are shared among mental disorders, especially
SCZ, bipolar disorder (BP) and ASD ${ }^{36,37}$. Abnormalities of brain morphology and function have been observed in SCZ, BP and ASD ${ }^{38-50}$, supporting aberrant neurodevelopment as the pathogenic mechanism of these disorders. The ZNF804A gene was first identified as a schizophrenia susceptibility gene at a genome-wide level ${ }^{51}$ and was subsequently reported to be associated with other psychiatric disorders such as bipolar disorder, autism spectrum disorder and heroin addiction ${ }^{6,22,51-54}$. As a transcription factor, ZNF804A may regulate neurodevelopment-related genes and therefore contributes to the pathogenesis of the disorders. Magnetic resonance imaging (MRI), cellular experiments and neurological data have shown that ZNF804A variants lead to brain structure and function changes ${ }^{26,55}$. The expression levels of genes that are involved in neurodevelopment and brain function such as SNAP25, NLGN4, COMT and $D R D 2$ are affected by $Z N F 804 A^{17,18,22}$. In this study, we identified a promoter SNP, rs10497655, associated with ASD. Subsequently, in vivo and in vitro experiments showed that this variant alters the binding affinity for the environmental-sensitive regulation factor HSF2, and influences the allelic expression levels of ZNF804A. To our knowledge, this was the first experiment to elucidate the process in which a disease-associated SNP in the ZNF804A gene affects the level of ZNF804A expression by binding with the upstream regulation factor HSF2. Our findings provide new insights into the role of ZNF804A polymorphisms in the pathogenesis of psychiatric disorders.

Our findings support that ZNF804A is a risk gene for ASD in Han Chinese populations. The newly identified functional SNP (rs10497655) alters the binding affinity to for an the environmental- sensitive regulation factor HSF2, and influences allelic expression levels of $Z N F 804 A$. We provide the new evidence that ZNF804A genetic variants contribute to the susceptibility to ASD.

\footnotetext{
Acknowledgements

This work was supported by the grants from National Key Special Projects of China (2016YFC0906400) to X.-H.G., grants from National Basic Research Program (973) of China (2013CB945403), the National Natural Science Foundation of China (81430005 and 31521003) and the National Key Basic Research Program of China (2016YFC1000502) to H.-Y.W. as well as grants from SHSMU-ION Research Center for Brain Disorders (2015NKX005) and Shanghai Key Laboratory of Psychotic Disorders (13dz2260500) to Y.-S.D.

\section{Author details}

'Department of Child \& Adolescent Psychiatry, Shanghai Mental Health Center, Shanghai Jiao Tong University School of Medicine, Shanghai 200030, China. ${ }^{2}$ Obstetrics and Gynecology Hospital, State Key Laboratory of Genetic Engineering at School of Life Sciences, Institute of Reproduction and Development, Fudan University, Shanghai 200011, China. ${ }^{3}$ Ministry of Education (MOE) Key Laboratory of Contemporary Anthropology, School of Life Sciences, Fudan University, Shanghai 200438, China. ${ }^{4}$ Department of Neurosurgery, Tianjin Children's Hospital, Tianjin 300134, China. ${ }^{5}$ Key Laboratory of Reproduction Regulation of NPFPC, Collaborative Innovation Center of Genetics and Development, Fudan University, Shanghai 200032, China. ${ }^{6}$ Children's Hospital of Fudan University, Shanghai 201102, China
} 


\section{Conflict of interest}

The authors declare that they have no conflict of interest.

\section{Publisher's note}

Springer Nature remains neutral with regard to jurisdictional claims in published maps and institutional affiliations.

Supplementary Information accompanies this paper at (https://doi.org/ 10.1038/s41398-019-0369-x).

Received: 20 July 2018 Revised: 10 December 2018 Accepted: 2 January 2019

Published online: 22 January 2019

\section{References}

1. AAmerican Psychiatric Association. Diagnostic and Statistical Manual of Mental Disorders: DSM-V 4189 (American Psychiatric Association, 2013), 4189-4189pp.

2. Baio, J. Prevalence of autism spectrum disorder among children aged 8 years autism and developmental disabilities monitoring network, 11 sites, United States, 2010. Morb. Mortal. Wkly. Rep. Surveill. Summ. 63, 1 (2015).

3. Ronald, A. \& Hoekstra, R. A. Autism spectrum disorders and autistic traits: a decade of new twin studies t. Am. J. Med. Genet. Part B Neuropsychiatr. Genet. 156, 255-274 (2011).

4. Yin, J. \& Schaaf, C. P. Autism genetics - an overview. Prenat. Diagn 37, 14-30 (2016).

5. Weiss, L. A., Arking, D. E., Daly, M. J. \& Chakravarti, A. A genome-wide linkage and association scan reveals novel loci for autism. Nature 461, 802-808 (2009).

6. Anney, R. et al. A genome-wide scan for common alleles affecting risk for autism. Hum. Mol. Genet. 19, 4072-4082 (2010).

7. Sanders, S. J. et al. De novo mutations revealed by whole-exome sequencing are strongly associated with autism. Nature 485, 237-241 (2012).

8. O'Roak, B. J. et al. Multiplex targeted sequencing identifies recurrently mutated genes in autism spectrum disorders. Science 338, 1619-1622 (2012).

9. Noroozi, R. et al. Glutamate receptor, metabotropic 7 (GRM7) gene variations and susceptibility to autism: a case-control study. Autism Res. Off. J. Int. Soc. Autism Res. 9, 1161-1168 (2016).

10. Landini, M. et al. Association analysis of noncoding variants in neuroligins 3 and $4 \mathrm{X}$ genes with autism spectrum disorder in an italian cohort. Int. J. Mol. Sci. 17, 1765 (2016).

11. Haghiri, R., Mashayekhi, F., Bidabadi, E. \& Salehi, Z. Analysis of methionine synthase (rs1805087) gene polymorphism in autism patients in Northern Iran. Acta Neurobiol. Exp. (Wars.) 76, 318-323 (2016).

12. Kondapalli, K. C. et al. Functional evaluation of autism-associated mutations in NHE9. Nat. Commun. 4, 2510 (2013).

13. Liu, S. et al. A rare variant identified within the GluN2B C-terminus in a patient with autism affects NMDA receptor surface expression and spine density. J. Neurosci. 37, 4093 (2017).

14. Pinto, D. et al. Functional impact of global rare copy number variation in autism spectrum disorders. Nature 466, 368-372 (2010).

15. Glessner, J. T. et al. Autism genome-wide copy number variation reveals ubiquitin and neuronal genes. Nature 459, 569-573 (2009).

16. Penzes, P., Cahill, M. E., Jones, K. A., VanLeeuwen, J. E. \& Woolfrey, K. M. Dendritic spine pathology in neuropsychiatric disorders. Nat. Neurosci. 14, 285-293 (2011).

17. Deans, P. J. et al. Psychosis Risk Candidate ZNF804A Localizes to Synapses and Regulates Neurite Formation and Dendritic Spine Structure. Biol. Psychiatry 82, 49-61 (2017).

18. Girgenti, M. J., LoTurco, J. J., Maher, B. J. ZNF804a Regulates Expression of the Schizophrenia-Associated Genes PRSS16, COMT, PDE4B, and DRD2. Plos ONE 7, e32404 (2012).

19. Braun, N. N., Reutiman, T. J., Lee, S., Folsom, T. D. \& Fatemi, S. H. Expression of phosphodiesterase 4 is altered in the brains of subjects with autism. Neuroreport 18, 1841-1844 (2007).

20. Hettinger, J. A., et al. DRD2 and PPP1R1B (DARPP-32) polymorphisms independently confer increased risk for autism spectrum disorders and additively predict affected status in male-only affected sib-pair families. Behav. Brain Funct. 8, 19 (2012).
21. Eicher, J. D. \& Gruen, J. R. Language impairment and dyslexia genes influence language skills in children with autism spectrum disorders. Autism Res. 8, 229-234 (2015).

22. Anitha, A. et al. Zinc finger protein 804A (ZNF804A) and verbal deficits in individuals with autism. J. Psychiatry Neurosci. 39, 294-303 (2014).

23. Griswold, A. J. et al. Evaluation of copy number variations reveals novel candidate genes in autism spectrum disorder-associated pathways. Hum. Mol. Genet. 21, 3513-3523 (2012).

24. Talkowski, M. E., et al. Sequencing chromosomal abnormalities reveals neurodevelopmental loci that confer risk across diagnostic boundaries. Cell 149, 525-537 (2012)

25. Steinberg, S. et al. Expanding the range of ZNF804A variants conferring risk of psychosis. Mol. Psychiatry 16, 59-66 (2011).

26. Chang, H., Xiao, X. \& Li, M. The schizophrenia risk gene ZNF804A: clinical associations, biological mechanisms and neuronal functions. Mol. Psychiatry 22, 944-953 (2017).

27. Li, M. et al. Allelic differences between Han Chinese and Europeans for functional variants in ZNF804A and their association with schizophrenia. Am. J. Psychiatry 168, 1318 (2011).

28. Hill, M. J. \& Bray, N. J. Evidence that schizophrenia risk variation in the ZNF804A gene exerts its effects during fetal brain development. Am. J. Psychiatry 169 1301-1308 (2012).

29. Ahn, S. G., Liu, P. C., Klyachko, K., Morimoto, R. I. \& Thiele, D. J. The loop domain of heat shock transcription factor 1 dictates DNA-binding specificity and responses to heat stress. Genes \& Dev. 15, 2134 (2001).

30. Anckar, J. \& Sistonen, L. Heat Shock Factor 1 as a Coordinator of Stress and Developmental Pathways 78-88 (Springer, New York, 2007).

31. Sandqvist, A. et al. Heterotrimerization of heat-shock factors 1 and 2 provides a transcriptional switch in response to distinct stimuli. Mol. Biol. Cell. 20, 1340-1347 (2009).

32. Akerfelt, M., Trouillet, D., Mezger, V. \& Sistonen, L. Heat shock factors at a crossroad between stress and development. Ann. N. Y. Acad. Sci. 1113, 15 (2007).

33. Umeda-Yano, S. et al. The regulation of gene expression involved in TGF- $\beta$ signaling by ZNF804A, a risk gene for schizophrenia. Schizophr. Res. 146, 273-278 (2013).

34. Baig, D. N., Yanagawa, T. \& Tabuchi, K. Distortion of the normal function of synaptic cell adhesion molecules by genetic variants as a risk for autism spectrum disorders. Brain Res. Bull. 129, 82-90 (2017).

35. Chang, E. H. et al. Postnatal neurodevelopmental expression and glutamatedependent regulation of the ZNF804A rodent homologue. Schizophr. Res. 168, 402-410 (2015).

36. Carroll, L. S., Owen, M. J. Genetic overlap between autism, schizophrenia and bipolar disorder. Genome Med. 1, 102 (2009).

37. Khanzada, N. S., Butler, M. G., Manzardo, A. M. Geneanalytics pathway analysis and genetic overlap among autism spectrum disorder, bipolar disorder and schizophrenia. Int. J. Mol. Sci. 18, E527 (2017).

38. Esslinger, C. et al. Neural mechanisms of a genome-wide supported psychosis variant. Science 324, 605-605 (2009).

39. Walter, H. et al. Effects of a genome-wide supported psychosis risk variant on neural activation during a theory-of-mind task. Mol. Psychiatry 16, 462 (2011).

40. Lencz, T. et al. A schizophrenia risk gene, ZNF804A, influences neuroanatomical and neurocognitive phenotypes. Neuropsychopharmacology 35 , 2284-2291 (2010).

41. Donohoe, G. et al. ZNF804A risk allele is associated with relatively intact gray matter volume in patients with schizophrenia. Neuroimage 54, 2132-2137 (2011).

42. Wei, Q. et al. ZNF804A rs1344706 is associated with cortical thickness, surface area, and cortical volume of the unmedicated first episode schizophrenia and healthy controls. Am. J. Med. Genet. Part B: Neuropsychiatr. Genet. 168, 265-273 (2015).

43. Alamian, G. et al. Alterations of intrinsic brain connectivity patterns in depression and bipolar disorders: a critical assessment of magnetoencephalography-based evidence. Front. Psychiatry 8, 41 (2017).

44. Mathew, I. et al. Medial temporal lobe structures and hippocampal subfields in psychotic disorders: findings from the Bipolar-Schizophrenia Network on Intermediate Phenotypes (B-SNIP) study. Jama Psychiatry 71, 769 (2014).

45. Meda, S. A. et al. Frequency-specific neural signatures of spontaneous lowfrequency resting state fluctuations in psychosis: evidence from Bipolarschizophrenia Network On Intermediate Phenotypes (B-SNIP) Consortium. Schizophr. Bull. 41, 1336-1348 (2015). 
46. Ivleva, E. I. et al. Gray matter volume as an intermediate phenotype for psychosis: Bipolar-Schizophrenia Network on Intermediate Phenotypes (B-SNIP). Am. J. Psychiatry 170, 1285-1296 (2013).

47. SJMC, Palmen, Engeland, Van, Hof, H., Schmitz, P. R. \& Neuropathological, C findings in autism. Brain A J. Neurol. 127(Pt 12), 2572 (2004).

48. Schumann, C. M., Barnes, C. C., Lord, C. \& Courchesne, E. Amygdala enlargement in toddlers with autism related to severity of social and communication impairments. Biol. Psychiatry 66, 942-949 (2009).

49. Cheung, C. et al. White matter fractional anisotrophy differences and correlates of diagnostic symptoms in autism. J. Child Psychol. Psyc 50, 1102-1112 (2009).

50. Anagnostou E., Taylor M. J. Review of neuroimaging in autism spectrum disorders: what have we learned and where we go from here. Mol. Autism 2, 4 (2011).
51. O'Donovan, M. C. et al. Identification of loci associated with schizophrenia by genome-wide association and follow-up. Nat. Genet. 40, 1053-1055 (2008).

52. Williams, H. J. et al. Fine mapping of ZNF804A and genome-wide significant evidence for its involvement in schizophrenia and bipolar disorder. Mol. Psychiatry 16, 429-441 (2011).

53. Zhang, C. et al. ZNF804A genetic variation confers risk to bipolar disorder. Mol. Neurobiol. 53, 2936-2943 (2016).

54. Sun, $Y$. et al. ZNF804A variants confer risk for heroin addiction and affect decision making and gray matter volume in heroin abusers. Addict. Biol. 21, 657-666 (2016).

55. Gurung, R. \& Prata, D. P. What is the impact of genome-wide supported risk variants for schizophrenia and bipolar disorder on brain structure and function? A systematic review. Psychol. Med. 45, 2461-2480 (2015). 